\title{
Application of the Monte Carlo Simulation in evaluating unconventional gas well economy
}

\author{
Wang Nan ${ }^{1,2}$, Zhao Qun ${ }^{1,2}$, Guo Wei ${ }^{1,2}$, Zang Huanrong ${ }^{1,2}$, Liu Dexun ${ }^{1,2}$ \\ ${ }^{1}$ Research Institute of Petroleum Exploration \& Development-LangFang, Hebei 065007, China \\ ${ }^{2}$ National Energy Research Institute of Petroleum Exploration and Development, PetroChina, Beijing \\ 065007, China.
} Keywords: Unconventional gas; Monte Carlo; index optimization; economic evaluation; decline
analysis; gas price; sensitivity analysis

\begin{abstract}
This paper starts with constructing the optimal process of shale gas economic indexes through selecting suitable statistics and prediction methods to confirm the major evaluation parameters of shale gas, such as single well recoverable reserves, single well composite cost and gas price. Then, economic benefit of shale gas single well projects was evaluated. Monte Carlo simulation was used to determine the probability distribution of the indexes that influence economic evaluation of single well projects. The sensitive factors that can influence financial internal return rate and net present value of shale gas single well projects were quantitatively analyzed. The correlations between impact factors and indexes were calculated and sorted. Finally, feasibility and risk level of single well projects were confirmed. This study determined the main factors that affect the economic performance of shale gas single well, evaluated the feasibility of shale gas single well projects, which can provide theoretical support for effectively controlling shale gas well cost, and reasonably and commercially developing shale gas reservoirs.
\end{abstract}

\section{Introduction}

Economic analysis of shale gas in China should be started with confirming evaluation parameters and related indexes of shale gas projects. In this paper, we think that single well productivity, single well composite cost and gas price within operation periods of such projects of shale gas wells are the three major evaluation parameters in optimal process of multiple indexes of shale gas projects.

\section{Identify parameters}

Single well productivity has positive relationships with horizontal interval lengths and amount of staged fracturing segments. Several methods can be used to predict single well controlled reserves within production cycle. Numerical simulation method can be utilized to establish numerical model of single shale gas well. After confirming some related parameters (components of shale gas, porosity, permeability and horizontal interval lengths, etc.), numerical simulation can be applied to calculate the estimated ultimate recoverable reserves (EUR). As shale gas exploration and production started late and production periods are short, there are no enough historical production data to do decline fitting analysis. Therefore, numerical simulation method was utilized in this paper to predict EUR of single well. The calculated EUR in Well Q1 is $1.05 \times 10^{8} \mathrm{~m}^{3}$.

While calculating single well cost, statistics method can be used to calculate the costs of predrilling engineering, drilling engineering, completion engineering and operation. In 2015, drilling and producing cost of shale gas wells in the United States was about US\$2.50-5.50 million (RMB 16-35 million). Shale gas development in China is still at initial stage, thus shale gas well costs are generally higher (around RMB 65-80 million investment for one horizontal well). With the improvement of engineering techniques and popularization of industrialized operation, the drilling and completion costs of shale gas in China can be decreased significantly. During middle and late development periods of shale gas fields, the costs of shale gas development may be similar to that of horizontal wells in the United States. 
Shale gas price is another important evaluation parameter. Considering the state subsidies for shale gas and distribution scope of natural gas prices, the final set gas price is RMB $1.31 / \mathrm{m}^{3}$, and the subsidized price is RMB $0.3 / \mathrm{m}^{3}$ during 2016-2017 and RMB $0.2 / \mathrm{m}^{3}$ during 2018-2020. These data were used in Monte Carlo simulation.

\section{Monte Carlo simulation}

\subsection{Monte Carlo simulation method}

While evaluating financial indexes of the project, because of multiple influence factors and complex calculation, general evaluation methods cannot fully reflect the impact of various uncertainties in the project construction process. Monte Carlo simulation can be used to more correctly reflect the impact of various uncertainties on project economic benefit. Monte Carlo simulation is also called random sampling or statistical testing method. It is based on probability and statistics theory, according to the process of calculation model, and takes the results of simulation experiments as the approximate solution of the question.

\subsection{Confirming parameter and variable distribution}

The uncertain factors in the process of project financial evaluation are usually considered as the uncertain parameters. By using the Monte Carlo simulation, we can analyze random variables to these uncertain parameters. After calculating various evaluation indexes of the project, we began to use the Monte Carlo simulation to analyze the uncertain factors that can influence financial indexes. The first step is to ascertain probability distribution of risk variables, which is a key step in the Monte Carlo simulation. The values of load factors that can influence probability distribution must consider related factors, such as gas field productivity, macro policy of the government, market risk and foreign experiences, etc.

In this paper, we used expert investigation method and empirical estimation method to fit the distribution of empirical data, assuming that single well composite cost and shale gas commodity rate are normal distribution. Other factors (natural gas price, single well production, operation cost and shale gas subsidy, etc.) are triangle distributions.

\subsection{Function analysis model}

In calculation, the variables and various evaluation parameters whose probability distributions have been confirmed should be input to the function analysis model, so as to construct the functional relations between these variables and index parameters. It is utilized to do 10000 times of stochastic numerical simulation to this project. The simulation results are listed in Table 1. The mean values, standard deviations, variances and variation coefficients etc. of FIRR and NPV are obtained.

Table 1 Results of Monte Carlo simulation

\begin{tabular}{c|c|c|c}
\hline Simulation prediction & IRR & Simulation prediction & IRR \\
\hline Simulation times & 10,000 & Variation coefficient & 1.15 \\
\hline Mean & $4.8 \%$ & Min & $-7.98 \%$ \\
\hline Median & $4.06 \%$ & Max & $41.01 \%$ \\
\hline Standard deviation & $5.47 \%$ & Mean error & $0.06 \%$ \\
\hline Variance & $0.9 \%$ & P10 & $4.05 \%$ \\
\hline Skewness & 0.98 & P50 & $4.41 \%$ \\
\hline Kurtosis & 5.0 & P90 & $6.05 \%$ \\
\hline
\end{tabular}


Calculation shows the mean FIRR is $4.8 \%$ in single well project, the skewness and kurtosis show normal distributions. When FIRR is 0 , the area at left side of the probability distribution graph accounts for $75.72 \%$ of the total area, and the right side is $24.28 \%$. According to the simulation results to the project NPV, the mean NPV is RMB -12.01 million, and the skewness and kurtosis show normal distributions. The probability of NPV $<0$ is $84.22 \%$, and that of NPV $\geqslant 0$ is only $15.78 \%$. When NPV is 0, the area at left side of the probability distribution graph accounts for $84.22 \%$ of the total area, and the right side is $15.78 \%$. The probability of higher NPV is lower for single well investment of shale gas, with higher investment risk.

According to the economic evaluation results of the project, there is some economic benefit in single well investment of shale gas. The FIRR (9.28\%) of the project is higher than the minimum FIRR (8\%) of unconventional oil and gas project for major oil companies in China. However, after quantifying several investment risk factors, the simulation results of two major evaluation indexes (FIRR and NPV) show that there is less profit possibility for shale gas project, with low economic benefit and higher investment risk.

\section{Analysis of uncertainties}

\subsection{Risk identification}

There are many uncertainties in the production of shale gas projects. There are many reasons for such uncertainties, including natural gas price changes, shale gas production, project investment cost, technique innovation, change of market mechanism, etc. Gas price is usually one of the most sensitive factors influencing project profit. To predict oil and gas prices and estimate price scope during the project operation period can greatly reduce the sensitivity of natural gas price and decrease the operation risk of shale gas projects.

Single well productivity of shale gas is another uncertain factor. Initial production, EUR scale and gas production rate change all directly control economic benefit of shale gas. Based on optimistic estimate, the initial production of shale gas wells is $15 \times 10^{4} \mathrm{~m}^{3} /$ day, EUR values of horizontal wells will be more than $1 \times 10^{8} \mathrm{~m}^{3}$, and $50-80 \%$ EUR can be produced from shale gas wells during initial 3-5 years. According to pessimistic estimate, the initial production of shale gas wells is $8 \times 10^{4} \mathrm{~m}^{3} /$ day, EUR value of horizontal wells will be more than $0.7 \times 10^{8} \mathrm{~m}^{3}$.

The increases of single well composite cost and operation cost can originate from intrinsic and extrinsic factors. The intrinsic factors include various treatment costs from increasing single shale gas well production and assuring stable production of shale gas wells, such as material and power costs of some technology measures. The extrinsic factors include some macroeconomic factors (such as currency inflation and tax rate) that can increase prices of raw materials. I

\subsection{Quantitative analysis}

After risk identification and qualitative analysis of the project, quantitative risk analysis should be carried out to the identified influence factors. In this paper, we adopted multi factor quantitative analysis method to calculate the changes of multi variables by calculation model. According to statistics results, the weight of gas price is the biggest $(35.4 \%)$, the weights of production and composite cost of single well are $30.2 \%$ and $-15.9 \%$. Gas price has the biggest influence on NPV of single well project.

Factors were sorted by their sensitivities on evaluation results. By sensitivity analysis, the risk variables that have the biggest influence on FIRR and NPV index parameters were selected. Among them, there are six variables whose influence factors on project are more than 0.2 . These factors have similar impact and rank on both FIRR and NPV. The gas price during project operation, single well production and shale gas subsidy price have positive relationships with project FIRR and NPV, with 
higher correlation coefficients $(0.55,0.50$ and 0.23 on FIRR; $0.60,0.53$ and 0.22 on NPV). Composite cost of single well, operation cost and exploration cost have negative relationship with FIRR, with correlation coefficients of $-0.36,-0.26$ and -0.19 , respectively. they have similar negative relationship with NPV, with correlation coefficients of $-0.43,-0.24,-0.22$ The rest five factors (benchmark yield, etc.) have influence factors less than 0.2, with lower sensitivities on FIRR and NPV.

\section{Conclusions}

Through Monte Carlo simulation, we obtained the following conclusions: Shale gas development projects have very big risk and uncertainty. An effective method of avoiding risks is to do economic benefit evaluation and enhance risk analysis.

Traditional economic evaluation methods have theory deficiency. Economic analysis based on Monte Carlo simulation can be used to determine the uncertainties and change laws of the influence factors, so as to reduce investment decision-making risk and improve scientific quality of project decision-making.

Shale gas exploration and development is a type of system engineering with long cycle, high risk, high investment and high payback. Under the current situation of low oil and gas prices, high gas well investment cost and no important breakthrough in shale gas well productivity, we propose to prudently invest in shale gas.

\section{References}

[1] Fagerstone. Exploratory Prospect Evaluation by Monte Carlo Simulation, 6085-MS SPE. (1976)

[2] Hasan A. AL-Ahmadi, Anas M. Almarzooq. Application of Linear Flow analysis to shale gas wells .-Field Cases. SPE 130370. (2010)

[3] James A. Murtha. Monte Carlo Simulation: Its Status and Future, SPE-37932-JPT.. (1997)

[4] James Li, C Mike Du. Vritical evaluation of shale gas reservoir simulation approaches: Single-porosity and dual-porosity modeling. SPE 141756. (2011)

[5] Jiehui Yuan, Dongkun Luo, Lianyong Feng. A review of the technical and economic evaluation techniques for shale gas development. Applied Energy. Volume 148, 15 June 2015, Pages 49-65.(2015)

[6] Jim, Murtha. Some Challenges for Monte Carlo Simulation. SPE-0206-013-TWA. . (2006)

[7] Luo Zuoxian. Shale Gas Exploration and Development in USA Today and Influence.[J]. Sino-Global Energy, 17(1):23-25. (2012)

[8] Mitch R. Withers, Robert Malina, et al.. Economic and environmental assessment of liquefied natural gas as a supplemental aircraft fuel. Progress in Aerospace Sciences, Volume 66, Pages 17-36. (2014)

[9] Nathaniel Heck, Courtney Smith, Eric Hittinger. A Monte Carlo approach to integrating uncertainty into the levelized cost of electricity.The Electricity Journal, Volume 29, Issue 3, Pages 21-30.(2016)

[10] Qian Xingkun, Jiang Xuefeng, et al.. Report on development of oil and gas industry at domestic and abroad in 2014. Beijing: CNPC Economics \& Technology Research Institute. (2015)

[11] Wang Nan, Liu Xinyuan, et al.. Lessons from U.S. and Canadian shale gas industry policies . International Petroleum Economics, 20(9) : 69-73. (2007) 